\title{
Solitary Bone Cysts of the Mandible: Two Case Reports and a Review of Literature
}

Arati S Neeli, SM Kotrashetti, Archana Louis

\begin{abstract}
Background: The solitary bone cyst is an uncommon nonepithelial cyst. Clinically, the lesion is asymptomatic in the majority of cases and is often accidentally discovered on routine radiological examination, frequently during the second decade of life. Its pathogenesis is still not clearly understood. The majority of solitary bone cysts are located in the mandibular body. This article presents two case reports, one in whom a cyst was diagnosed on routine radiographic examination and other patient reported with dull ache and radiographic examination showed bilateral presentation of the cystic lesion.
\end{abstract}

Materials and methods: In both patients the biopsy was planned. On surgical opening the lesions presented empty cavities. Curettage was done and overlying bone sent for histopathological examination. Both patients were followed for 1 year.

Results: At follow-up visit both patients were examined clinically and radiographically. In both the patients resolution of the lesion was seen at follow-up visit.

Conclusion: Solitary bone cyst is a rare entity with typical clinical and radiographic features. However, confirmation of diagnosis can be made only by histological examination. Solitary bone cyst may be secondary to an underlying bone pathology. Solitary bone cyst of the head and neck may vary in presentation and severity. Surgical removal is the treatment of choice and provides a satisfying outcome.

Keywords: Bone cysts, Solitary bone cyst, Mandible.

How to cite this article: Neeli AS, Kotrashetti SM, Louis A. Solitary Bone Cysts of the Mandible: Two Case Reports and a Review of Literature. World J Dent 2013;4(3):193-197.

Source of support: Nil

Conflict of interest: None declared

\section{INTRODUCTION}

Solitary bone cysts (SBCs) are benign neoformations which can affect all the skeletal bones. Over $90 \%$ of solitary bone cysts are located in long bones most commonly the proximal humerus and femur. Of the less than $10 \%$ that are found in the gnathic bones, the mandible is favored over three quarters of the time (75\%). SBCs are uncommon, representing $1 \%$ of all jaw cysts. ${ }^{1}$ In 1929 , Lucas and Blum for the first time described solitary bone cyst as a separate disease entity. ${ }^{2}$ However, it was not until 1946 that the diagnostic criteria of these cysts was established by Rushton. ${ }^{3}$ The lesion is mainly diagnosed in young patients most frequently during the second decade of life. Clinically, the lesion is asymptomatic in the majority of cases and is often accidentally discovered on routine radiological examination, usually as an unilocular radioluscent area with a 'scalloping effect' and either lacking contents or containing liquid and or connective tissue. SBCs frequently occur during the second decade of life and majority are located in the mandibular body. Its pathogenesis is still not clearly understood SBCs have been reported in the literature under a variety of names: traumatic bone cyst, hemorrhagic bone cyst, extravasation cyst, progressive bone cavity, simple bone cyst and unicameral bone cyst when it occurs in long bones. ${ }^{4-8}$ The multitude of the names applied to this lesion attests to the lack of understanding of the true etiology and pathogenesis. Different causal factors have been proposed: bone tumor degeneration, altered calcium metabolism, low-grade infection, local alterations in bone growth, venous obstruction, increased osteolysis, intramedullary bleeding, local ischemia, or a combination of such factors. ${ }^{3,7}$ It has been suggested that any form of trauma, including tooth extraction could give rise to a cyst of this kind. ${ }^{9}$ Although, it is important to underline that the SBCs' etiology is unknown. Following are the documented cases of solitary bone cyst in the body of the mandible, of unknown etiology followed by a brief review of literature of the main characteristics of the lesion.

\section{CASE REPORTS}

\section{Case 1}

A 11-year-old girl, presented to the Department of Orthodontics with a chief complaint of forwardly placed upper front teeth. She had symmetric face with slightly forwardly placed upper anteriors (Fig. 1). As a part of treatment planning, the patient was advised an OPG. On the OPG a irregular radiolucency with interdental scalloping was seen extending from 32 to 36. Radiographically, it looked like cystic lesion with trabaculae passing through it. Lower border of the mandible was resorbed (Fig. 2). The patient gave no history of any trauma and neither did she have offending tooth. All the teeth were vital. Aspiration of the lesion yielded reddish serosanguinous fluid. Thus, a differential diagnosis of solitary bone cyst, aneurismal bone cyst and hemangioma was made. The patient was prepared for biopsy in order to arrive at a final diagnosis. Vestibular incision was made. A buccal mucoperiosteal flap was raised. 
A window was created in the buccal cortical bone and the cavity was exposed. On exposure, a cavity extending from midline (incisors) to first molar was seen with some trabeculae extending from the margins into the defect (Fig. 3). The intraoperative presentation of the lesion was typical of a solitary bone cyst. The cavity was thoroughly curetted and bleeding was induced into the lesion. The flap was sutured back and the patient was recalled for follow-up after 7 days. Bone specimen was sent for histopathological examination. The biopsy report confirmed diagnosis of solitary bone cyst. Postoperative recovery was uneventful. At follow-up period clinical and radiographic examination showed evidence of normal healing (Figs 4 and 5). The patient was followed-up for a period of 1 year.

\section{Case 2}

A 40-year-old female patient (Fig. 6) presented to our institution with a complaint of dull intermittent pain in the left region of the jaw since 6 months. On examination, there was no offending teeth in that region. All the teeth in that quadrant were vital. General clinical examination revealed no abnormality. An OPG was advised to rule out any pathology. OPG (Fig. 7) revealed two multiloculated radiolucent lesions on either side of the jaw in relation to premolars and molars. On aspiration, both the cavities yielded serous fluid.

Routine hematological investigations were normal. Biopsy was planned. Bilateral buccal flaps were raised. Overlying bone was removed on both sides which revealed empty cavities with some connective tissue within, which was curetted out (Figs 8 and 9). Connective tissue and the overlying bone was sent for histopathology. A diagnosis of bilateral solitary bone cyst was made. This patient was followed for 1 year. OPG showed evidence of bone regeneration in the lesion (Fig. 10).

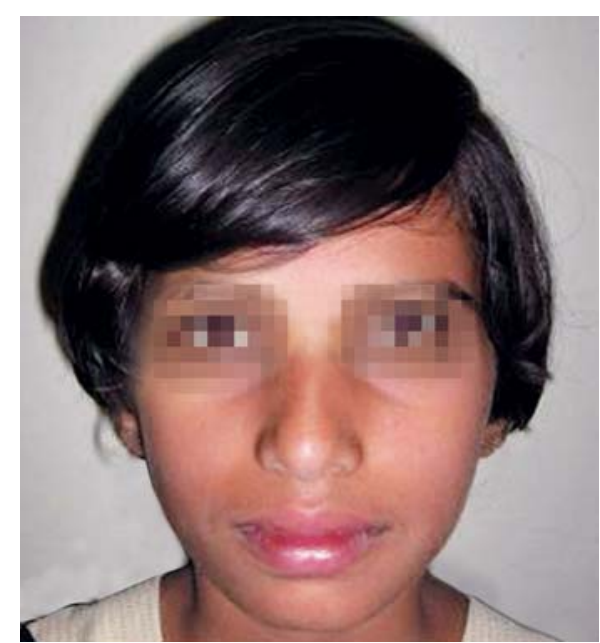

Fig. 1: Case 1 having symmetric face with no swelling on the affected side

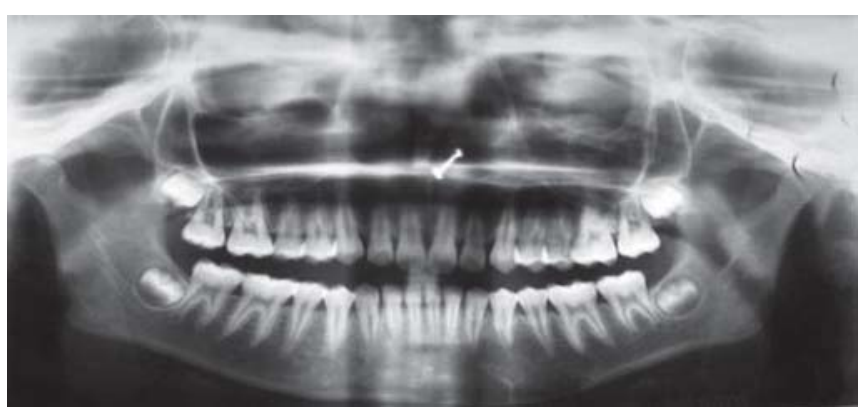

Fig. 2: OPG showing radiolucent lesion in the mandibular body from 32 to 36

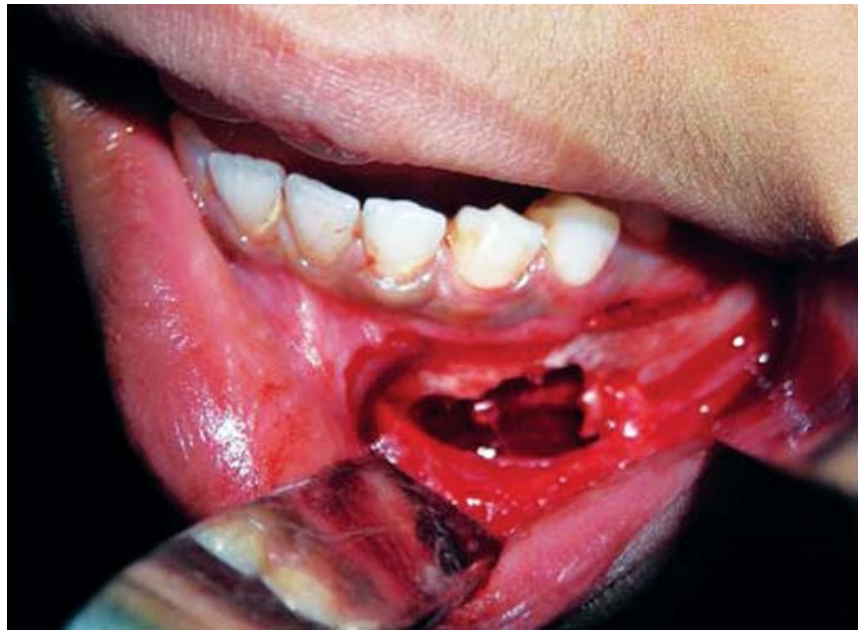

Fig. 3: Intraoperative photograph showing empty cavity on the left mandibular body

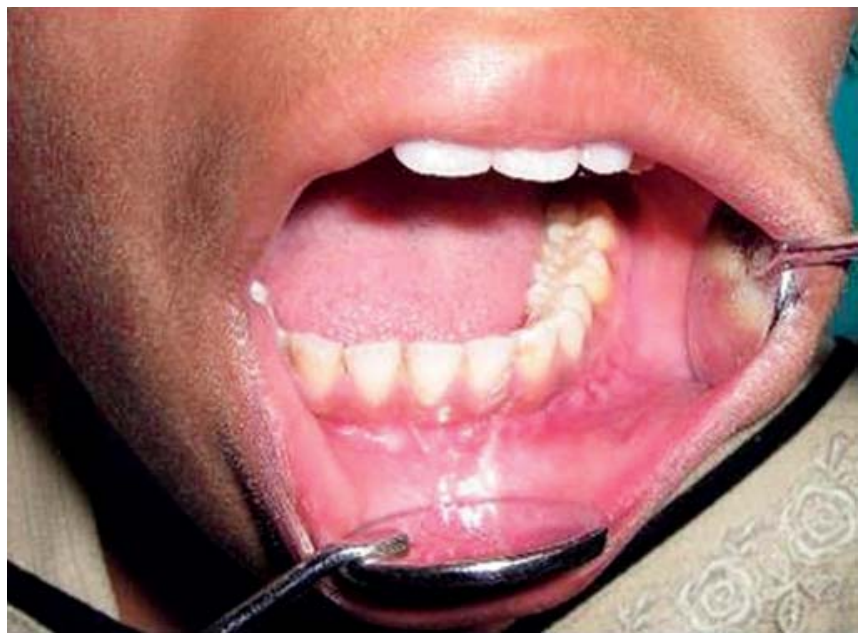

Fig. 4: Clinical photograph after 10 days

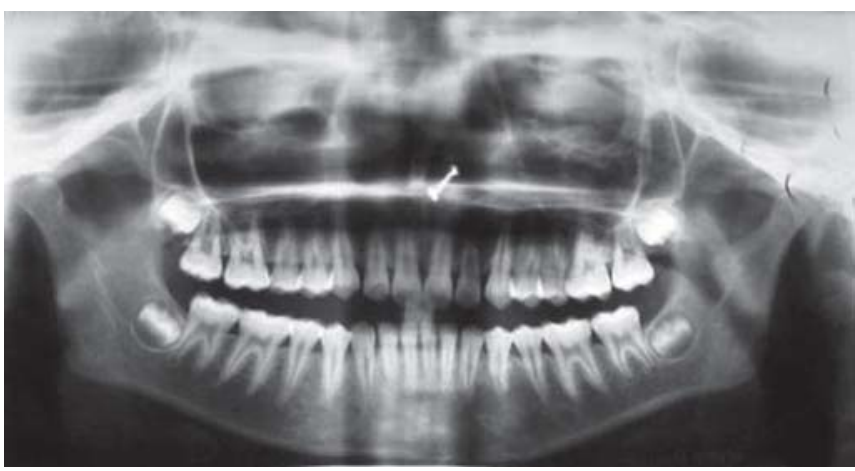

Fig. 5: OPG at 6-month follow-up showing bone regeneration 


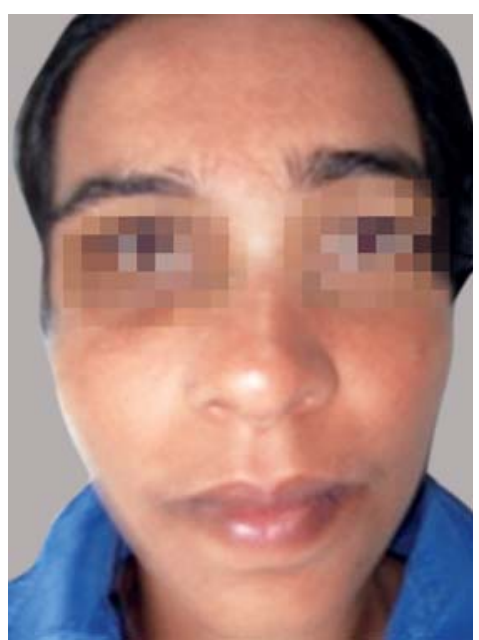

Fig. 6: Case 2 having no obvious extraoral manifestation

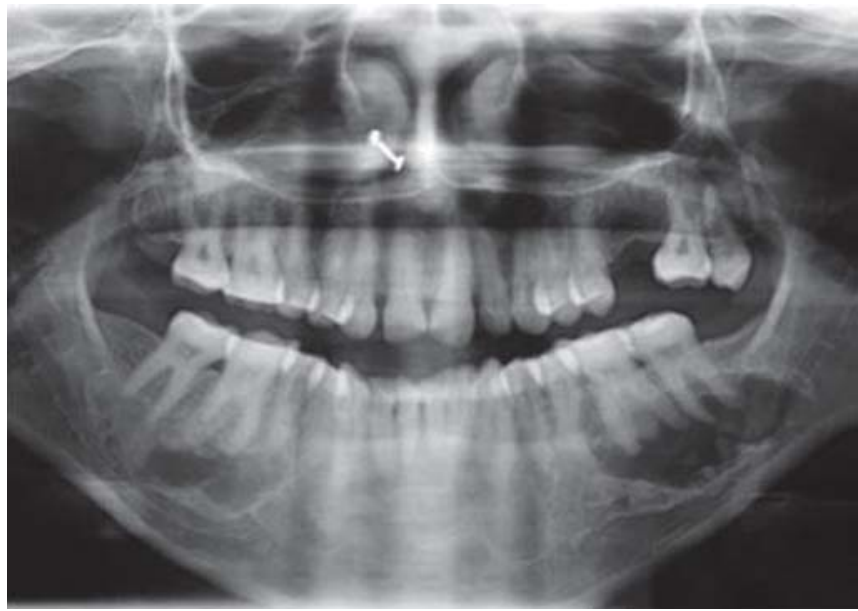

Fig. 7: OPG showing bilateral radiolucent lesion in the molar area

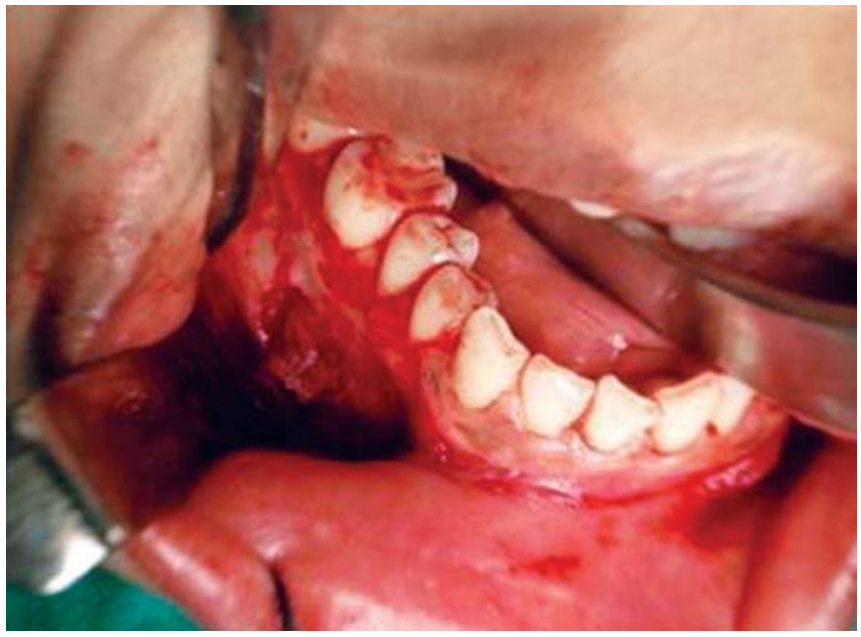

Fig. 8: Intraoperative photograph showing empty cavity on the right side

\section{DISCUSSION}

Solitary bone cyst of the jaws is uncommon, representing approximately $1 \%$ of all jaw cysts. The first case presented by us is a 11-year-old girl with a solitary bone cyst present

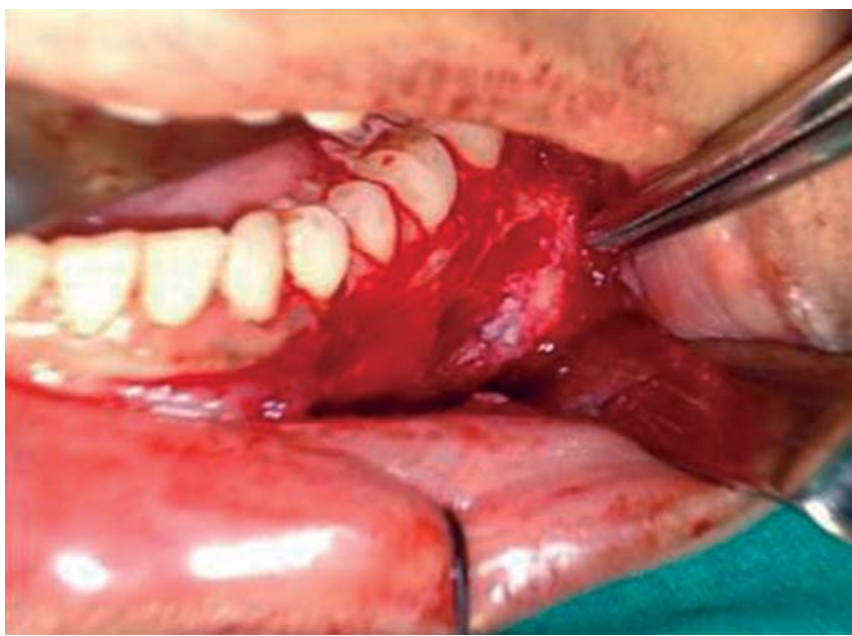

Fig. 9: Intraoperative photograph showing empty cavity on the left side

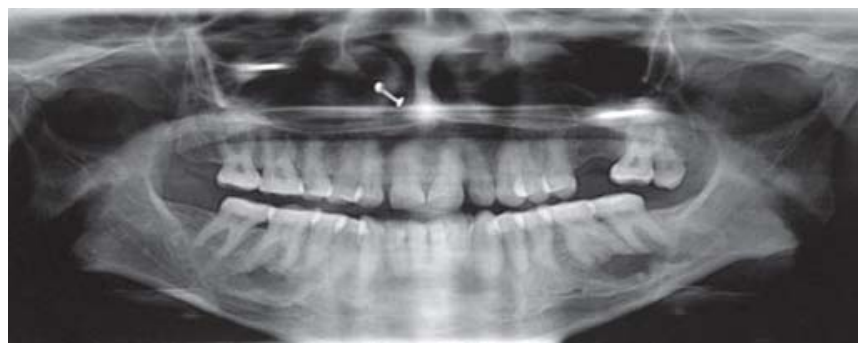

Fig. 10: Postoperative X-ray after 1 year showing bilateral bone regeneration

in the left body of the mandible. These findings are in accordance with the reports by Kaugars and Cale, who said that solitary bone cysts have an equal prevalence in both genders, $75 \%$ occur in first 2 decades of life (mean age of 13 years), and most often (75\%) affect the posterior mandible, predominantly the body region. ${ }^{1,10}$ However, studies by Peñarrocha et $\mathrm{al}^{11}$ and Ballester et al ${ }^{12}$ show a clear female predilection.

Reviews of the literature show that simple bone cysts of the jaws no longer can be considered rare. Second case showed bilateral presentation of the lesion. There have been very few cases of bilateral involvement reported previously. ${ }^{13-16}$ The clinical and radiographic appearances are mostly rather typical, but in some cases there are some variations such as occurence in older age group, can be symptomatic and may appear in different regions such as ramus or condyle. ${ }^{8,17}$ Age of second patient was 40 years and complained of dull ache associated with lesion on one side and asymptomatic on the other side.

The etiology of the cyst in both the cases is not known. Various hypotheses have been proposed for the pathogenesis of the traumatic bone cyst. The most frequently proposed theory for the development of these lesions involves a traumatic event inciting medullary hemorrhage and a 
subsequent failure of the hematoma to organize and be replaced with tissue. ${ }^{13}$ The time interval between the trauma and the discovery of a traumatic bone cyst (TBC) varies in the literature from 1 week to 20 years. ${ }^{3,18}$ Howe and Jacobs supported the theory that the content of the cavity depends on the length of time that the cyst has existed. ${ }^{3,19}$ When discovered early, the lesion usually contains blood or serosanguineous fluid. The amount of fluid diminishes with the age of the lesion and eventually becomes empty. Blum and Toma believed that there must also be a predisposing idiosyncratic factor in the pathogenesis of SBC, such as a peculiarity of the vessel wall or an abnormal coagulation of the blood. ${ }^{19,20}$ Beasley believed that the histological changes observed in their cases of SBCs tended to support the picture of a degenerative process of vascular or neurogenic source of origin and supported the theory that injury or nerve damage within bone results in vascular ischemia and subsequent necrosis to an area. ${ }^{21}$

The location of the cyst in both these cases was in the body of the mandible. This is the most common site of occurrence for solitary bone cyst. ${ }^{1,3,18,22,23}$ Histopathological reports of both the cases showed many osteoblasts forming woven bone and multinucleated giant cells , fibrous tissue lining containing numerous endothelial lined blood vessels with deposits of hemosiderin. The radiographic, histopathological and operative findings of the cases are evident with the literature review. ${ }^{11,24}$

It has been suggested that SBCs may undergo spontaneous resolution. ${ }^{25}$ However, an expectant approach to management cannot be recommended, in view of the possibility of diagnostic error. Failure to provide treatment may lead to additional complications such as pathological mandibular fracture. ${ }^{26}$ In both patients, treatment consisted of careful curettage of the bone walls, with satisfactory results characterized by progressive bone regeneration and the absence of relapse.

Other alternative treatments, such as filling of the cavity with bovine lyophilized bone or the introduction of autologous blood with bone from the patient or hydroxyapatite, may be of interest in those cases where conventional management fails. ${ }^{27}$ However, the introduction of radiopaque materials in the cavity may complicate the diagnosis of possible lesion relapse.

\section{CONCLUSION}

Maxillofacial SBCs are uncommon lesions with that often respond to treatment by surgical curettage. Recurrence may be attributed to incomplete removal of the lesion. The solitary bone cyst is usually asymptomatic with occasional findings, and its etiology, is unknown in majority of the cases. The cavity is usually seen to be empty and without epithelial lining. Careful curettage of the lesion favors progressive bone regeneration, offering a good prognosis and an almost negligible relapse rate.

\section{REFERENCES}

1. Harnet JC, Lombardi T, Klewansky P, Rieger J, Tempe MH, Clavert JM. Solitary bone cyst of the jaws: a review of the etiopathogenic hypotheses. J Oral Maxillofac Surg 2008;66(11):2345-2348.

2. Lucas CD, Blum T. Do all cysts in the jaws originate from the dental system? J Am Dent Assoc 1929;16:647-661.

3. Rushton M. Solitary bone cysts in the mandible. Br Dent $\mathrm{J}$ 1946;81:37-49.

4. Howe GL. 'Haemorrhagic cysts' of the mandible. Br J Oral Surg 1965;3:55-91.

5. Boyne PJ. Treatment of extravasation cysts with freeze dried homogenous bone grafts. J Oral Surg 1956;14:206-212.

6. Whinery JG. Progressive bone cavities of the mandible. Oral Surg Oral Med Oral Pathol 1955;8:903-916.

7. Jaffe HL, Lichtenstein L. Solitary unicameral bone cyst. Arch Surg 1942;44:1004-1025.

8. Cowan CG. Traumatic bone cysts of the jaws and their presentation. Int J Oral Surg 1980;9:287-291.

9. Pogrel MA. A solitary bone cyst possibly caused by removal of an impacted third molar. J Oral Maxillofac Surg 1987;45: 721-723.

10. Kaugars GE, Cale AE. Traumatic bone cyst. Oral Surg Oral Med Oral Pathol 1987;63:318.

11. Peñarrocha-Diago M, Sanchis-Bielsa JM, Bonet-Marco J, Minguez- Sanz JM. Surgical treatment and follow-up of solitary bone cyst of the mandible: a report of seven cases. Br J Oral Maxillofac Surg 2001;39:221-223.

12. Cortell-Ballester I, Figueiredo R, Berini-Aytés L, Gay-Escoda C. Traumatic bone cyst: a retrospective study of 21 cases. Med Oral Patol Oral Cir Bucal 2009 May 1;14(5):E239-243.

13. Heimdahl A. An unusual case of simple bone cyst of the mandible. Int J Oral Surg 1978 Feb;7(1):32-35.

14. Markus AF. Bilateral haemorrhagic bone cysts of the mandible: A case report. Br J Oral Surg 1979 Mar;16(3):270-273.

15. Patrikiou A, Sepheriadou-Mavropoulou T, Zambelis G. Bilateral traumatic bone cyst of the mandible. a case report. Oral Surg Oral Med Oral Pathol 1981;51(2):131-133.

16. Brannon RB, Houston GD. Bilateral traumatic bone cysts of the mandible: an unusual clinical presentation. Med Mil 1991 Jan;156(1):20-22.

17. Magliocca KR, Edwards SP, Helman JI. Traumatic bone cyst of the condylar region: report of 2 cases. J Oral Maxillofac Surg 2007;65:1247.

18. Huebner G, Turlington E. So-called traumatic (hemorrhagic) bone cysts of the jaws. Oral Surg 1971;31:354-365.

19. Thoma KH. A symposium on bone cysts (editorial). Oral Surg 1955;8:899-901.

20. Blum T. Additional report on traumatic bone cysts. Oral Surg Oral Med Oral Pathol 1955;8(9):917-939.

21. Beasley JD. Traumatic cyst of the jaws: report of 30 cases. J Am Dent Assoc 1955;92:145-152. 
22. MacDonald-Jankowski D. Traumatic bone cysts in the jaws of a Hong Kong Chinese population. Clinical Radiology 1995;50:787-791.

23. Kuttenberger J, Farmand M, Stoss H. Recurrence of a solitary bone cyst of the mandibular condyle in a bone graft. Oral Surg Oral Med Oral Pathol 1992;74:550-556.

24. Hansen LS, Sapone J, Sproat RC. Traumatic bone cysts of jaws. Oral Surg Oral Med Oral Pathol 1974;37:899-910.

25. Sapp JP, Stark ML. Self-healing traumatic bone cysts. Oral Surg Oral Med Oral Pathol 1990;69:597-602.

26. Hughes CL. Hemorrhagic bone cyst and pathologic fracture of mandible: report of case. J Oral Surg 1969;27:345-346.

27. Dellinger TM, Holder R, Livingston HM, Hill WJ. Alternative treatments for a traumatic bone cyst: A longitudinal case report. Quintessence Int 1998;29:497-550.

\section{ABOUT THE AUTHORS}

\section{Arati S Neeli (Corresponding Author)}

Reader, Department of Oral and Maxillofacial Surgery, KLE VK Institute of Dental Sciences, Belgaum, Karnataka, India e-mail: aratiaqua@yahoo.co.in

\section{SM Kotrashetti}

Professor and Head, Department of Oral and Maxillofacial Surgery KLE VK Institute of Dental Sciences, Belgaum, Karnataka, India

\section{Archana Louis}

Postgraduate Student, Department of Oral and Maxillofacial Surgery KLE VK Institute of Dental Sciences, Belgaum, Karnataka, India 